\title{
A high-throughput customized cytokinome screen of colon cancer cell responses to small-molecule oncology drugs
}

\author{
Kelsey E. Huntington ${ }^{1,2,3,4,5}$, Anna Louie ${ }^{1,2,3,6}$, Lanlan Zhou ${ }^{1,2,3,4}$ and Wafik S. El-Deiry ${ }^{1,2,3,4,5}$ \\ ${ }^{1}$ Laboratory of Translational Oncology and Experimental Cancer Therapeutics, Warren Alpert Medical School, Brown University, \\ Providence, RI 02912, USA \\ ${ }^{2}$ The Joint Program in Cancer Biology, Brown University and Lifespan Health System, Providence, RI 02912, USA \\ ${ }^{3}$ Cancer Center at Brown University, Warren Alpert Medical School, Brown University, Providence, RI 02912, USA \\ ${ }^{4}$ Department of Pathology and Laboratory Medicine, Warren Alpert Medical School, Brown University, Providence, RI 02912, \\ USA \\ ${ }^{5}$ Pathobiology Graduate Program, Brown University, Providence, RI 02912, USA \\ ${ }^{6}$ Department of Surgery, Brown University, Lifespan Health System and Warren, Alpert Medical School, Brown University, \\ Providence, RI 02912, USA \\ Correspondence to: Wafik S. El-Deiry, email: wafik@brown.edu \\ Keywords: cytokine profiling; inflammatory response; chemokine; growth factor; immune profiling \\ Received: August 07,2021 Accepted: September 03, $2021 \quad$ Published: September 28, 2021
}

Copyright: @ 2021 Huntington et al. This is an open access article distributed under the terms of the Creative Commons Attribution License (CC BY 3.0), which permits unrestricted use, distribution, and reproduction in any medium, provided the original author and source are credited.

\section{ABSTRACT}

Inflammatory cytokines, chemokines, and growth factors are molecular messengers that circulate and have the capability to modify the tumor microenvironment and impact therapeutic response. The characterization of soluble mediators as biomarkers for diagnosis and prognosis is of interest in oncology. We utilize the cytokinome to characterize the response of colorectal tumor cell lines to selected small-molecules in oncology as a proof-of-concept dataset with immunomodulatory analyte heat map rankings for drug and cell line combinations. We observed overall trends in drug class effects with MEK-, BRAF-, PARP-inhibitors, and Imipridones in cytokine, chemokine, and growth factor responses that may help guide therapy selection. MEK-inhibitor treatment downregulated analytes VEGF, CXCL9/MIG, and IL-8/CXCL8 and upregulated CXCL14/BRAK, Prolactin, and CCL5/ RANTES. BRAF-inhibitor treatment downregulated VEGF and IL-8/CXCL8, while increasing soluble TRAIL-R2. Treatment with PARP-inhibitors decreased CXCL9/MIG, IL-8/CXCL8, CCL3/MIP-1 alpha, VEGF, and CXCL14/BRAK, while treatment increased soluble TRAIL-R2 and prolactin. Treatment with Imipridones decreased CCL3/MIP-1 alpha, VEGF, CXCL14/BRAK, IL-8/CXCL8, and Prolactin and increased CXCL5/ENA-78. We also observed differential responses to therapeutics depending on the mutational profile of the cell line. In the future, a similar but larger dataset may be utilized in the clinic to aid in the prediction of patient response to immunomodulatory therapies based on tumor genotype.

\section{INTRODUCTION}

Cytokines, chemokines, and growth factors are all molecular messengers of the immune system that impact tumor behavior and host response. Cytokines are either secreted or membrane-bound proteins that regulate cellular signaling and can be categorized as pro- or anti-inflammatory. Chemokines are proteins that mediate chemotaxis in nearby cells and play an important role in the recruitment of either immunosuppressive or immunostimulatory cell types to the tumor microenvironment. Differential expression of chemokines regulates the selective migration of myriad cell types [1]. Growth factors are usually secreted proteins or steroid hormones that promote cell proliferation and differentiation. Each of these categories of immunomodulating agents are produced by both tumor and immune cells, among other cell types, and can impact therapeutic response [2, 3]. 
The characterization of these soluble mediators as biomarkers of both diagnosis and prognosis is a rapidly evolving topic in cancer research and clinical oncology. Biomarkers have been correlated with clinical outcome in several different tumor types including colorectal cancer (CRC) [4-6]. In CRC, differentially expressed plasma or serum cytokines represent potential biomarkers for diagnosis and prognosis. Yamaguchi et al. found that the levels of cytokines in plasma varied significantly between patients with CRC and control subjects [4]. However, cytokine signaling is highly pleotropic with one cytokine producing diverse and sometimes opposing effects depending on the signaling context [2]. Moreover, cytokine signaling is characterized by a high degree of redundancy where discrete cytokines produce the same functional effects [7]. The combination of pleotropic and redundant outcomes in response to a particular cytokine makes therapeutic manipulation challenging. Furthermore, there exists a degree of heterogeneity in the prognostic value of cytokines, with some showing opposing correlations in response to therapy across multiple tumor types $[5,8]$.

\section{RESULTS}

\section{Cytokinome profiling of human colorectal cancer cell lines with diverse mutations using a high- throughput custom multiplexed analyte panel}

Cell lines that represent diverse mutational backgrounds in tumor suppressor or oncogene drivers such as TP53, KRAS, BRAF, PIK3CA, APC, TRK, CTNNB1, BRCA2, TGFB2, and PTEN were selected for the analyses reported (Table 1). The three cell lines HCT-116, HT-29, and KM12C were included because they represent varied mutational profiles and were predicted to respond differently to differing therapeutic mechanisms of action. Importantly, we included both microsatellite stable (MSS) and microsatellite instability positive (MSI+) cell lines to observe how microsatellite status impacts response to small-molecule treatment, which has implications in combination with checkpoint blockade therapies.

Selected oncologic small-molecules with distinct mechanisms of action encompassed several classes of drugs such as PARP-, MEK-, and BRAF-inhibitors, among others (Table 2). We primarily selected FDAapproved small-molecules, but also included several experimental small-molecules in oncology that target commonly dysregulated pathways in cancer. We were especially interested in the results of the experimental drugs that are either currently in clinical trials or are planned for clinical trials in the near future (such as GSK-3 inhibitor 9-ING-41, and Imipridones ONC201, ONC206, and ONC212). The selected cell lines displayed a range of susceptibility to the drug panel (Figure 1, Supplementary Table 2).
We designed a high-throughput custom multiplex cytokine, chemokine, and growth factor profiling panel based on both pro- and anti-inflammatory markers, as well as cytokines and chemokines involved in the recruitment and activation of immune cells such as natural killer (NK) and T cells. Cell lines were treated with the drug panel and cell culture supernatants were analyzed using Luminex 200 technology (Figure 2). Cell lines were treated at differing concentrations (IC-10, IC-30, IC-50, IC-70, and IC-90) to determine dose-response effects for each smallmolecule. It is important to emphasize that the panel was designed to analyze soluble factors that are secreted or shed by tumor cells post-treatment with drug.

To analyze these results, we graphed dose-response values for each drug and cell line combination and calculated the linear regression. To generate heat maps that rank analytes for each drug and cell line combination from most-downregulated to most up-regulated, we utilized the slope of the linear regression. We then examined the top five most up- and down-regulated analytes from each heat map (Supplementary Table 1).

To better visualize the data, we created a summary heatmap that grouped cytokines, chemokines, and growth factors into two categories: (1) analytes that are correlated with immunosuppression or unfavorable prognosis $[6,9-18]$ or (2) analytes that are correlated with immunostimulation or favorable prognosis in the context of CRC, specifically [19-26] (Figure 3). When designing these two groups, we focused on the implications of a particular analyte when colon cancer cell-derived.

\section{Class effects on cytokine, chemokine, and growth factor profiles were observed across multiple human colorectal cancer cell lines}

There were several classes of drugs of which we tested more than one inhibitor and observed class effects. Drug classes where we tested multiple compounds included MEK inhibitors, BRAF inhibitors, PARP inhibitors, and Imipridones. For MEK inhibitors, we tested both Trametinib and Selumetinib, and saw similarities in the top five most-downregulated analytes in response to both drugs across all three cell lines (6 groups total) (Figure 4). We saw decreases in VEGF (6 out of 6), CXCL9/MIG (5 out of 6), and IL-8/CXCL8 ( 5 out of 6 ). The analytes that most notably increased after treatment with MEK inhibitors in all cell lines were CXCL14/BRAK (4 out of 6), Prolactin (4 out of 6), and CCL5/RANTES (4 out of 6). Next, for BRAF inhibitors Dabrafenib and Vemurafenib we again saw similar trends in response across all cell lines tested (Figure 5). We observed decreases in VEGF (6 out of 6), and IL-8/ CXCL8 (5 out of 6). By contrast, we observed increases in soluble TRAIL-R2 (sTRAIL-R2) (4 out of 6). Next, PARP inhibitors included Olaparib and Rucaparib and once again we observed notable decreases in VEGF (6 out of 6), 
Table 1: Mutational background of selected colon cancer cell lines

\begin{tabular}{|c|c|c|c|c|c|c|c|c|c|c|c|c|c|c|c|}
\hline $\begin{array}{l}\text { CRC Cell Line } \\
\text { Name }\end{array}$ & Species & $\begin{array}{c}\text { MSI/MSS } \\
\text { status }\end{array}$ & TP53 & HRAS & NRAS & KRAS & BRAF & PIK3CA & $\begin{array}{c}\text { PTEN } \\
\text { expression }\end{array}$ & APC & TRK & CTNNB1 & ACVR2A & BRCA2 & TGFBR2 \\
\hline HCT-116 & human & MSI & WT & WT & WT & MT & WT & MT & positive & WT & WT & MT & MT & MT & WT \\
\hline HT-29 & human & MSS & MT & WT & WT & WT & MT & MT & positive & MT & WT & WT & WT & WT & MT \\
\hline KM12C & human & MSI & MT & WT & WT & WT & WT & WT & null & MT & MT & UN & MT & MT & MT \\
\hline
\end{tabular}

Abbreviation: WT: wild type; MT: mutant; UN: unknown. HCT-116, HT-29, and KM12C colon cancer cell line mutational backgrounds.

CXCL9 (5 out of 6), IL-8 (5 out of 6), CCL3/MIP-1 alpha (4 out of 6), and CXCL14/BRAK (4 out of 6) (Figure $6)$. The analytes that increased as a class effect included sTRAIL-R2 (5 out of 6), and Prolactin (4 out of 6). Lastly, for Imipridones we tested three different compounds including ONC201, ONC206, and ONC212 (9 groups) (Figure 7). We saw notable decreases in VEGF (6 out of 9), CCL3/MIP-1 alpha (5 out of 9), CXCL14/BRAK (6 out of 9), IL-8/CXCL8 (6 out of 9), and Prolactin (5 out of 9). In contrast, we saw increases in CXCL5/ENA-78 (6 out of 9 ).

\section{Drug effects on cytokine, chemokine, and growth factor profiles were observed across multiple cell lines}

We also evaluated several drugs that belonged to additional classes of small-molecules (Supplementary
Figure 1). First, we looked at GSK-3 inhibitor 9-ING41 and saw decreases in VEGF (3 out of 3), CXCL9/ MIG (3 out of 3), and CCL3/MIP-1 alpha (2 out of 3). Meanwhile, we observed increases in CXCL14 (3 out of 3 ), IL-8/CXCL8 (2 out of 3), sTRAIL-R2 (3 out of 3), and sTRAIL-R3 (2 out of 3). Next, we focused on Crizotinib, a c-MET inhibitor, and saw decreases in VEGF ( 3 out of 3), CXCL9/MIG (2 out of 3), and CXCL13/BLC/ BCA-1 (2 out of 3 ). In contrast, we observed increases in IL-8/CXCL8 (2 out of 3), sTRAIL-R2 (2 out of 3), Prolactin (2 out of 3), and CXCL14 (2 out of 3). Next, we examined Dasatinib, a tyrosine kinase inhibitor, and observed decreases in IL-8/CXCL8 (3 out of 3), VEGF (2 out of 3), CXCL9/MIG, and CXCL5 (2 out of 3). In contrast, we noted increases in sTRAIL-R2 (3 out of 3 ), CXCL14/BRAK (3 out of 3), and Prolactin (2 out of 3). We next examined Duvelisib, a PI3K inhibitor and saw decreases in CCL3/MIP-1 alpha (2 out of 3), VEGF (3 out
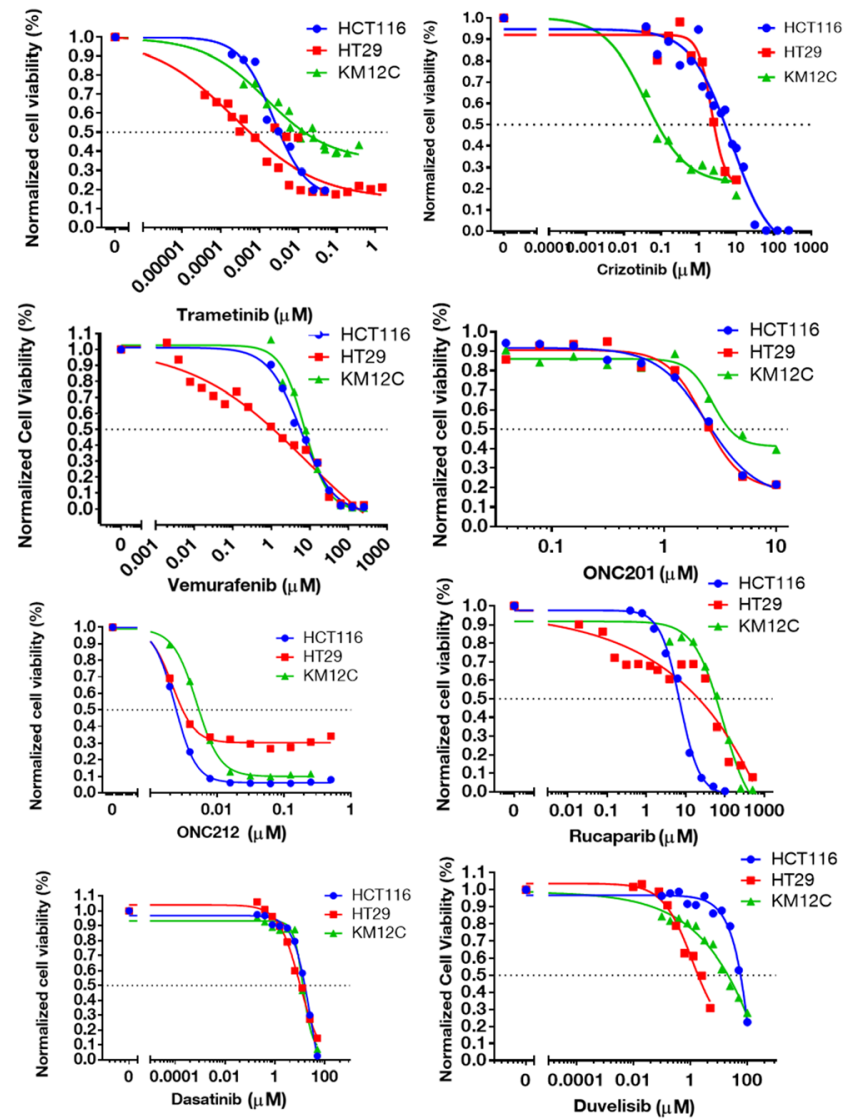
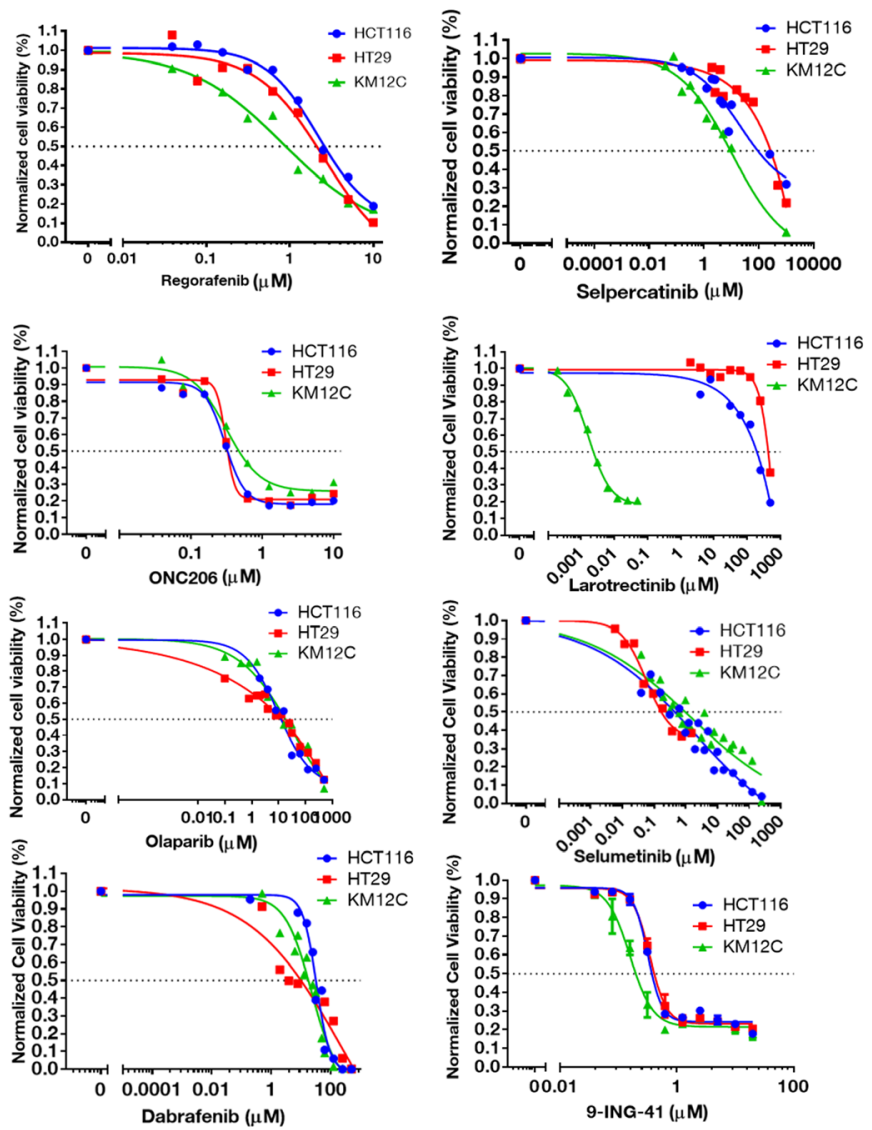

Figure 1: IC-50 curves for selected small-molecules in HCT-116, HT-29, and KM12C cell lines. Cell viability curves post 72-hour treatment were graphed in GraphPad and used to determine IC-50 values. 
of 3), sTRAIL-R2 (2 out of 3), IL-8/CXCL8 (2 out of 3), CXCL9/MIG (2 out of 3 ) and CXCL14/BRAK (2 out of 3 ). We observed an increase in Prolactin (2 out of 3 ). We next analyzed Larotrectinib, a TRK inhibitor, and noted decreases in VEGF (3 out of 3), CXCL9/MIG (3 out of 3 ), CCL3/MIP-1 alpha (2 out of 3), and IL-8/CXCL8 (2 out of $3)$. Meanwhile, we observed increases in CCL5/RANTES ( 2 out of 3 ), IL-18/IL-1F4 (2 out of 3 ), and Prolactin (2 out of 3 ). We also analyzed Regorafenib, a multikinase inhibitor, and again noted decreases in VEGF (3 out of 3 ), CXCL9/MIG (3 out of 3), IL-8/CXCL8 (3 out of 3), CCL3 (2 out of 3 ), and CXCL5/ENA-78 (2 out of 3). In contrast, we noted increases in CXCL14/BRAK (2 out of 3 ), and Prolactin (2 out of 3 ). We then examined RET inhibitor Selpercatinib, and observed decreases in VEGF (3 out of 3), CXCL14/BRAK (2 out of 3), CCL3/MIP-1 alpha (2 out of 3 ), and sTRAIL-R2 (2 out of 3). Lastly, we saw increases in IL-8/CXCL8 (2 out of 3), CXCL5/ENA-78 ( 3 out of 3 ), and Prolactin ( 2 out of 3 ).

\section{DISCUSSION}

The most commonly downregulated analyte in response to all treatment conditions was vascular
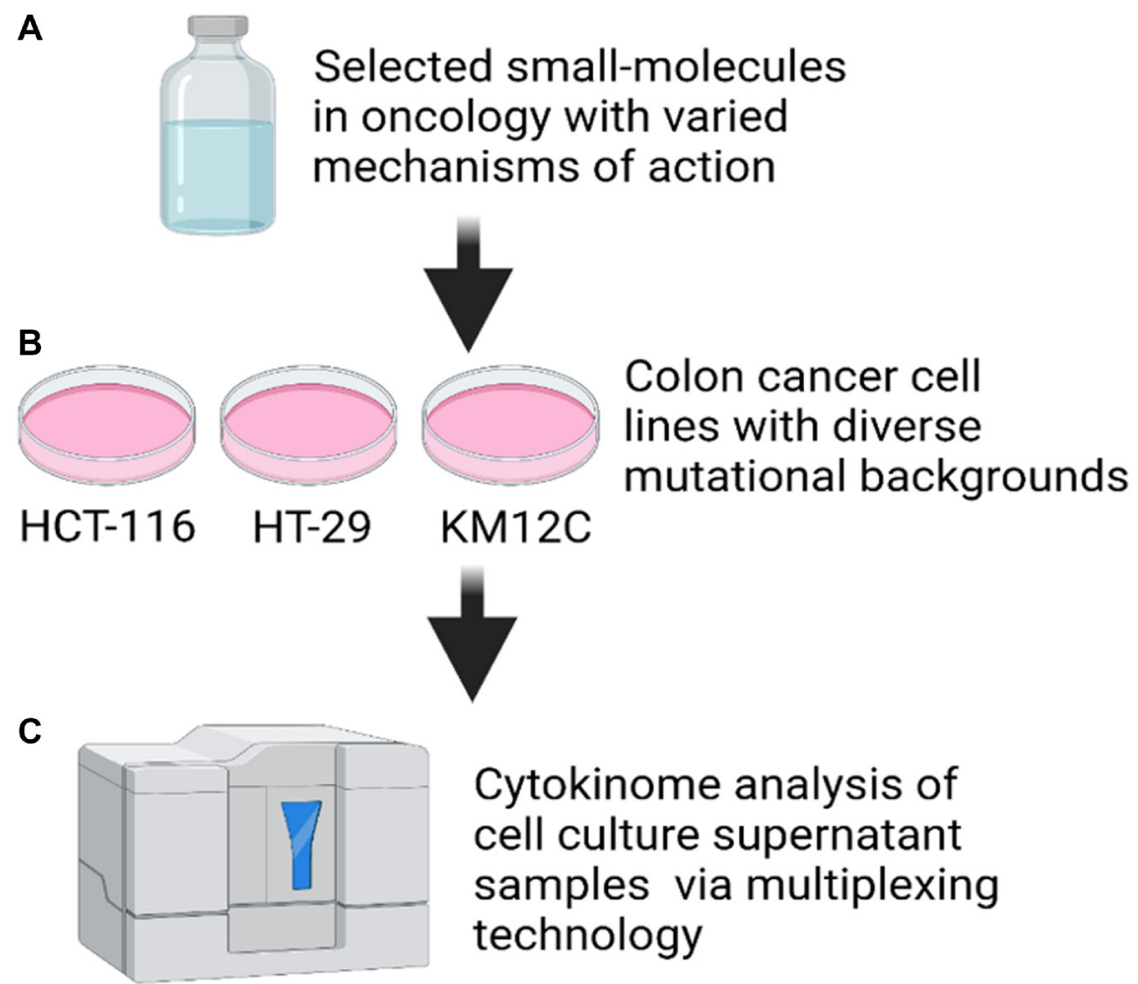

Cytokinome analysis of cell culture supernatant samples via multiplexing technology

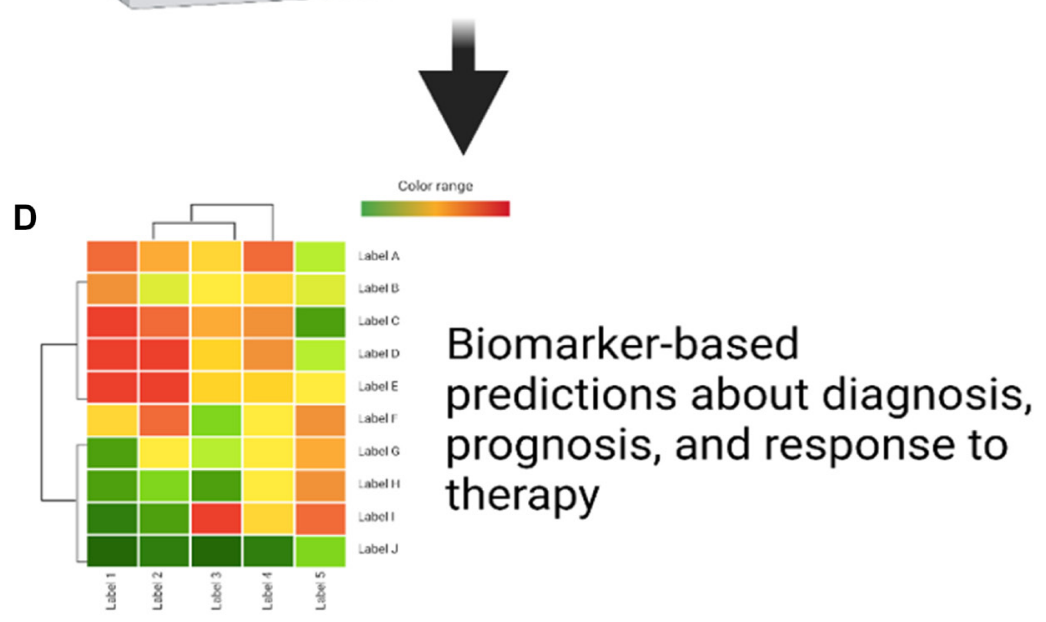

Figure 2: Cell culture supernatant cytokinome analysis workflow. (A) Small-molecules in oncology were selected to provide varied mechanisms of action. (B) Three colon cancer cell lines (HCT-116, HT-29, and KM12C) were selected based on differing mutational backgrounds in key tumor suppressor genes. (C) Cytokinome analysis was performed on cell culture supernatant samples after 48 hours of treatment using a Luminex 200. (D) Biomarker-based predictions about diagnosis, prognosis, and response to therapy were made based on heat maps generated from linear regression analysis of the data. 
Table 2: Drug classes of selected small-molecule drugs

\begin{tabular}{ll}
\hline Drug & Class \\
\hline Trametinib & MEK1/2 inhibitor \\
Crizotinib & Tyrosine kinase inhibitor \\
Larotrectinib & TRK inhibitor \\
Selpercatinib & RET inhibitor \\
Vemurafenib & BRAF inhibitor \\
Regorafenib & Tyrosine kinase inhibitor \\
ONC201 & Imipridone \\
ONC206 & Imipridone \\
ONC212 & Imipridone \\
Rucaparib & PARP inhibitor \\
Olaparib & PARP inhibitor \\
Selumetinib & MEK inhibitor \\
Dabrafenib & BRAF inhibitor \\
Dasatinib & Tyrosine kinase inhibitor \\
Duvelisib & PI3K inhibitor \\
9 -ING-41 & GSK-3 inhibitor \\
\hline
\end{tabular}

Drug name is listed in the first column and drug class is listed in the second column.

endothelial growth factor (VEGF). VEGF is an angiogenic factor that is upregulated in many cancer types, including $\mathrm{CRC}$, and promotes tumor angiogenesis. In $\mathrm{CRC}, \mathrm{VEGF}$ expression in tumor tissue and patient plasma samples correlates with disease progression and metastasis [27]. Moreover, VEGF-positive tumors [28], high postoperative plasma VEGF concentrations [29], and high serum VEGF levels are correlated with decreased overall survival in CRC [30]. The downregulation of VEGF that we observed as a common trend despite heterogenous cell line mutational profiles and therapeutic mechanisms of action could suggest the possibility of off-target or nonspecific effects. The identification of VEGF as a tumor cell-secreted marker that is commonly altered by smallmolecules will require further interrogation. Another commonly downregulated analyte was CXCL9/MIG, an

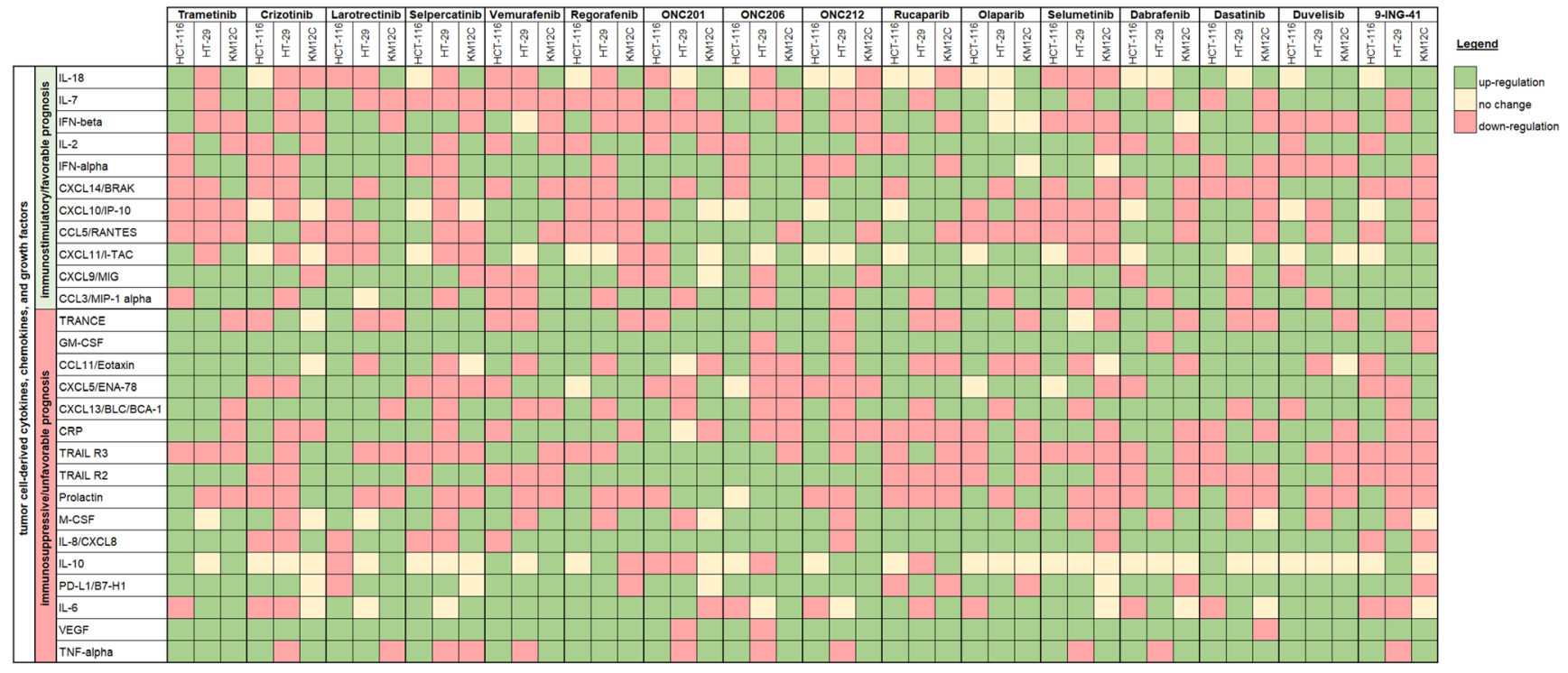

Figure 3: Immune synergy heat map showing cell line changes in cytokine, chemokine, and growth factor profiles in response to therapeutic treatment. Cytokines, chemokine, and growth factors are grouped into one of two categories: (1) analytes that are correlated with immunosuppression or unfavorable prognosis or (2) analytes that are correlated with immunostimulation or favorable prognosis in the context of CRC. The heat map is based on the slope of the linear regression where green indicates upregulation, yellow indicates no change, and red indicates downregulation post-treatment. 
important chemokine for both recruitment and activation of leukocytes mediated by binding to CXCR3, a receptor expressed on activated $\mathrm{T}$ cells. It has been shown that expression of CXCL9/MIG is higher in patients with colon cancer as compared to healthy controls [31]. Furthermore, CXCL9/MIG expression was corelated with
A
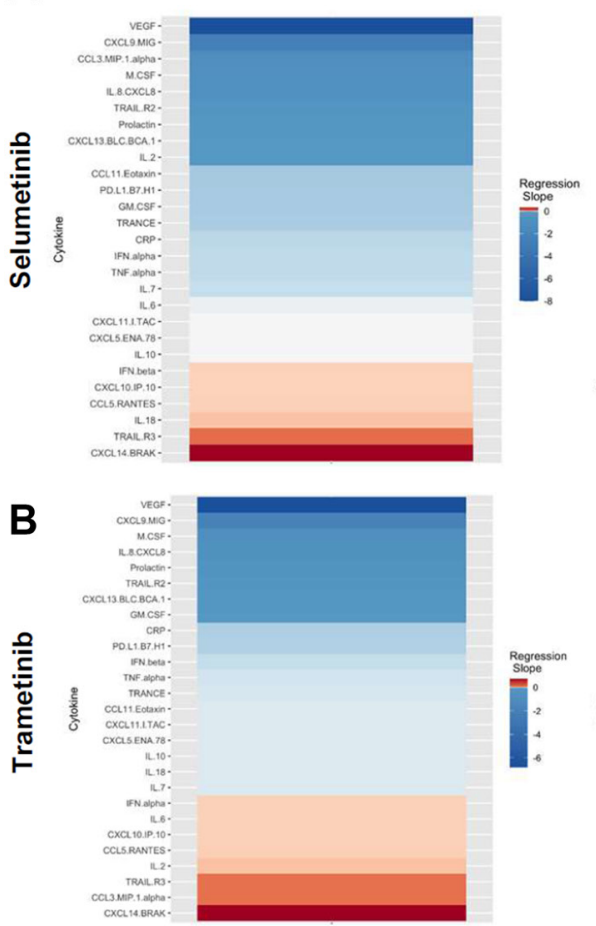

HT-29
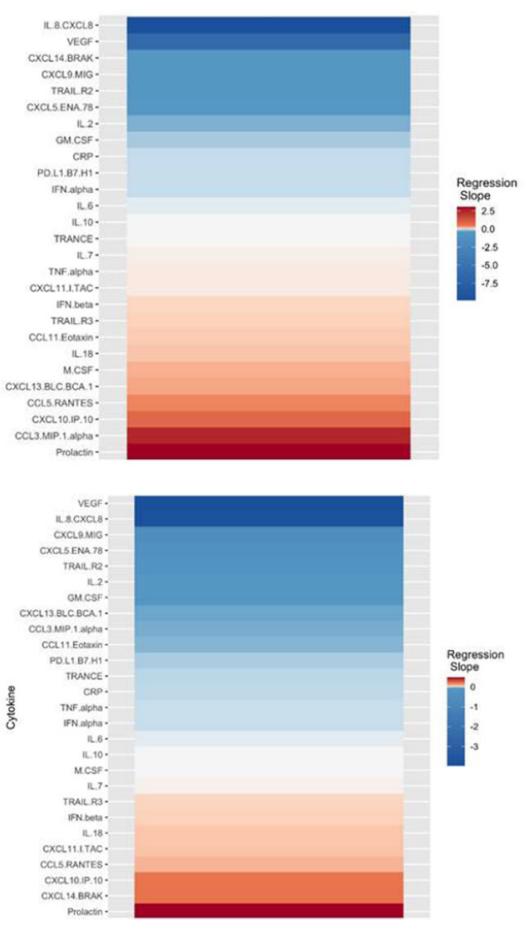

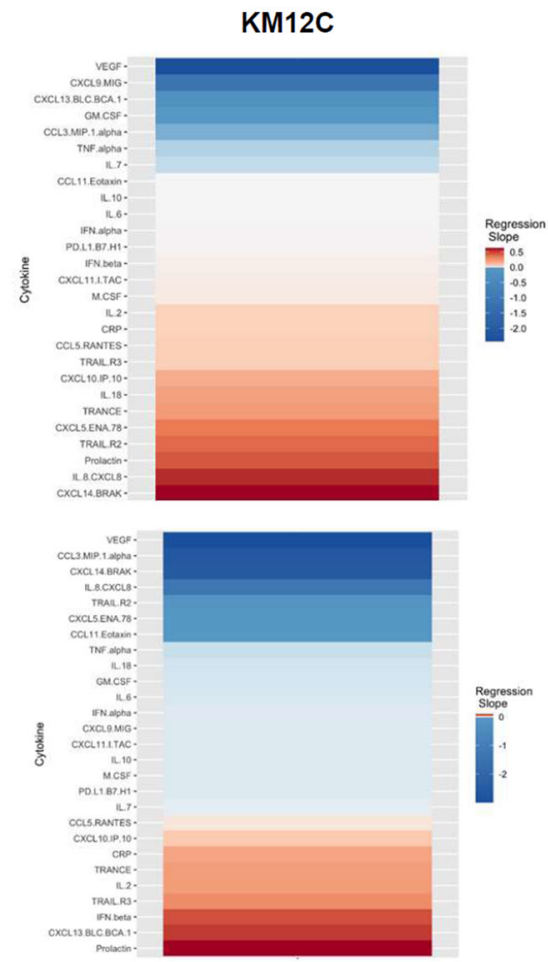

Figure 4: Heatmaps displaying regression slopes of cytokine profiles for MEK inhibitors. (A) Heat maps based on regression slopes for HCT-116, HT-29, and KM12C after 48-hour treatment of increasing doses of Selumetinib or (B) Trametinib.
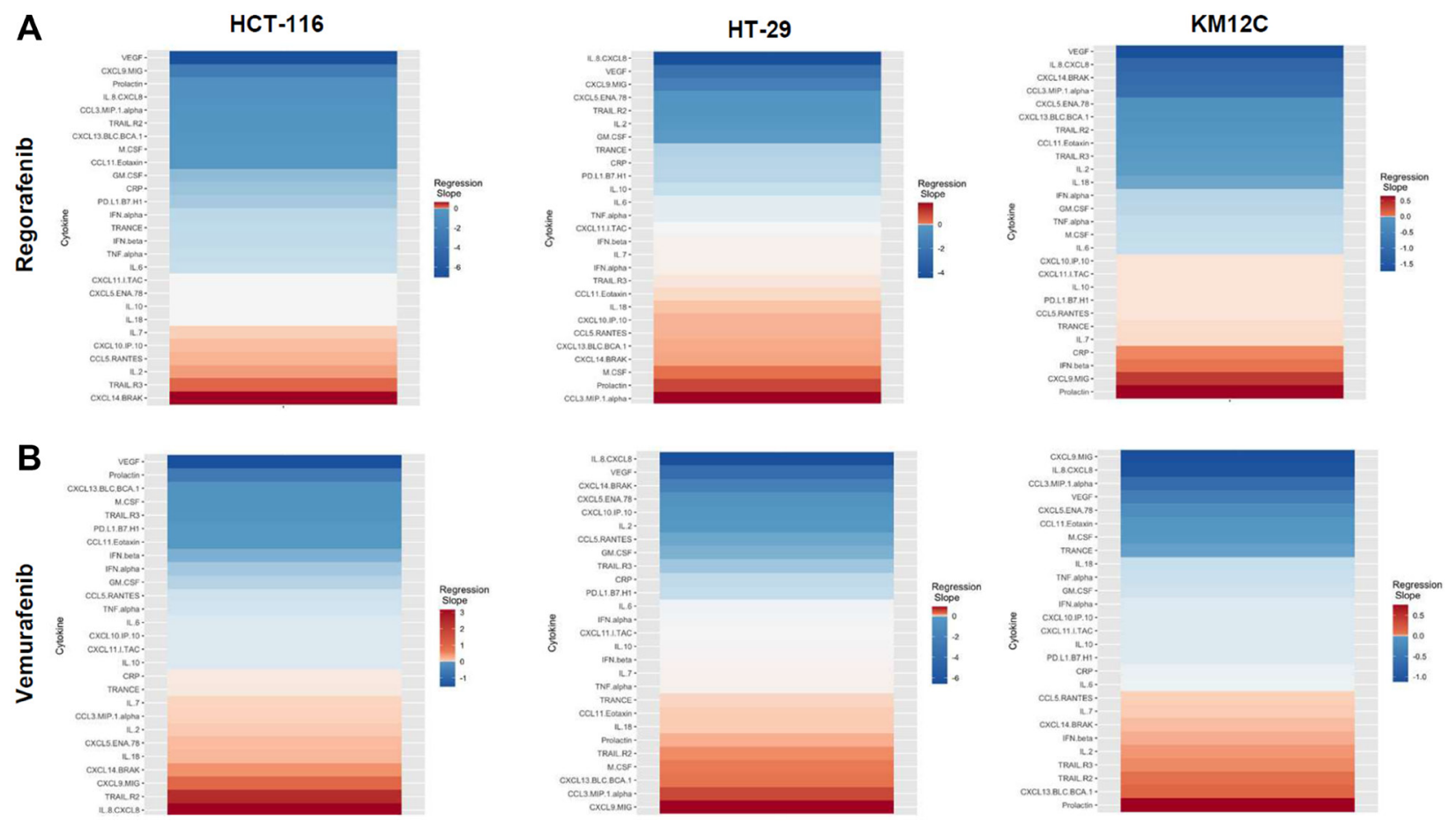

Figure 5: Heatmaps displaying regression slopes of cytokine profiles for BRAF inhibitors. (A) Heat maps based on regression slopes for HCT-116, HT-29, and KM12C after 48-hour treatment of increasing doses of Regorafenib or (B) Vemurafenib. 
the presence of tumor-infiltrating lymphocytes as well as post-operative survival [31]. Next, we saw recurrences in IL-8/CXCL8 down-regulation post-treatment with our drug panel. IL-8/CXCL8 expression is significantly associated with colorectal tumorigenesis and metastasis [32] and has been suggested as a therapeutic target for this reason. Moreover, it is known that IL-8/CXCL8 induces epithelial-mesenchymal transition (EMT) in tumor cells via the PI3K/Akt signaling axis [33]. Another chemokine commonly downregulated was CCL3/MIP-1 alpha, which plays an important role in lymphocyte recruitment, activation, proliferation, and differentiation in colon cancer murine models [34]. Lastly, we observed a common post-treatment decrease in CXCL14/BRAK, a small chemokine with controversial effects in tumorigenesis [35-37]. The clinical correlation of this biomarker with disease prognosis remains unclear at this time, as several have reported that elevated levels of CXCL14/BRAK expression in tumor sections correlates with worse overall survival [38], yet others have reported the opposite [39].

Interestingly, CXCL14/BRAK was also among one of the analytes most commonly upregulated. We also noted a common increase in the hormone prolactin, which is commonly overexpressed in patients with colorectal cancer [40]. Next, we observed a recurrent upregulation of CCL5/RANTES which is chemotactic for many leukocytes and plays an important role in immune cell recruitment to inflammatory sites. In contrast, the CCR5/CCL5 axis has also been reported to play a role in the proliferation, metastasis, and formation of an immunosuppressive microenvironment [41]. Tumorderived CCL5/RANTES has been shown to enhance regulatory $\mathrm{T}$ cell-mediated killing of cytotoxic $\mathrm{T}$ cells in colon cancer [42]. Moreover, CCL-5 deficiency has been shown to increase tumor infiltrating $\mathrm{CD} 8+\mathrm{T}$ cells in the context of CRC [43]. Lastly, we noted an upregulation of CXCL5/ENA-78 under many of the treatment conditions across several cell lines, which may induce colorectal cancer angiogenesis [44].

We also monitored soluble receptors TNF-related apoptosis-inducing ligand Receptor 2 (sTRAIL-R2)/ Death Receptor 5 (sDR5) and TNF-related apoptosisinducing ligand Receptor 3 (sTRAIL-R3). TRAIL-R2 is well-known as a cell surface receptor that triggers apoptosis upon binding with its cognate ligand, TNFrelated apoptosis-inducing ligand (TRAIL). In contrast, TRAIL-R3 is known as a decoy receptor for TRAIL, as it lacks a cytoplasmic death domain rendering it unable to induce apoptosis. The soluble versions of these receptors presumably both function as decoy receptors
A

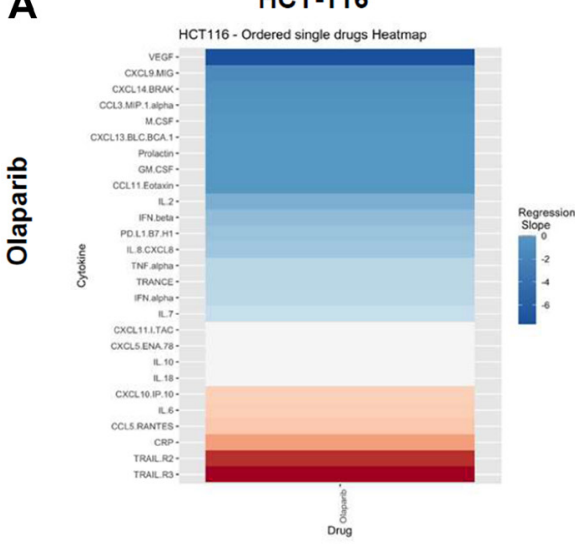

B

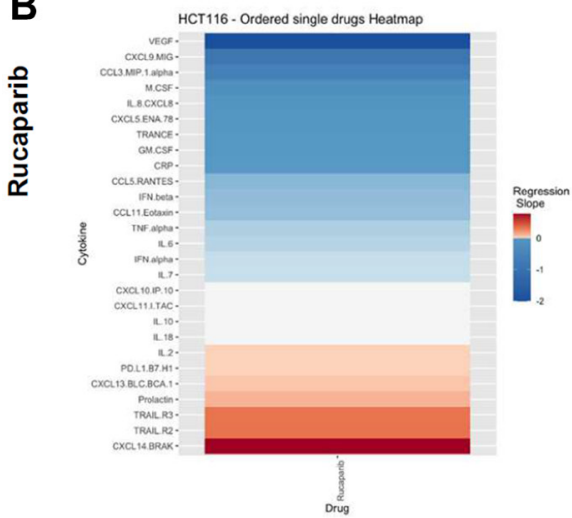

HT-29
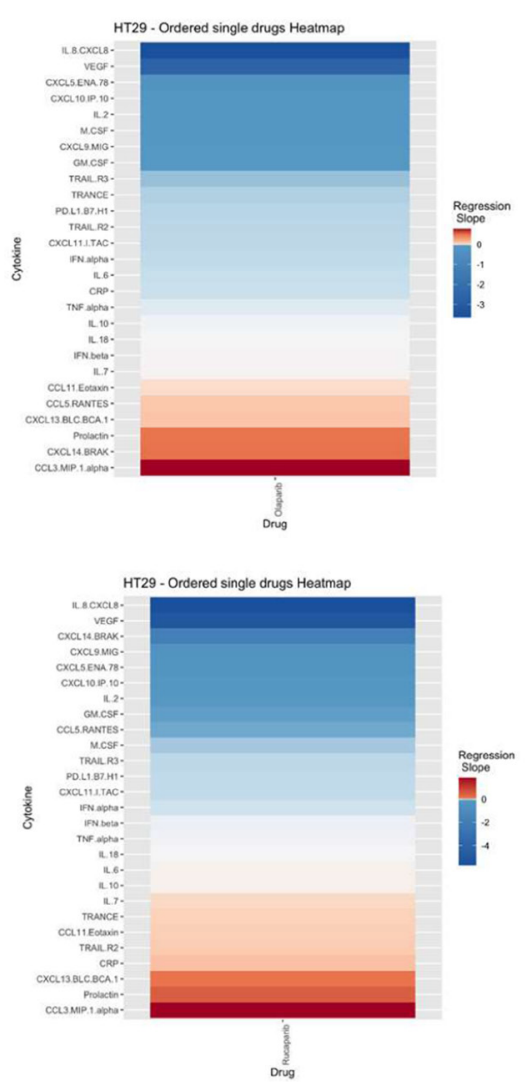

KM12C
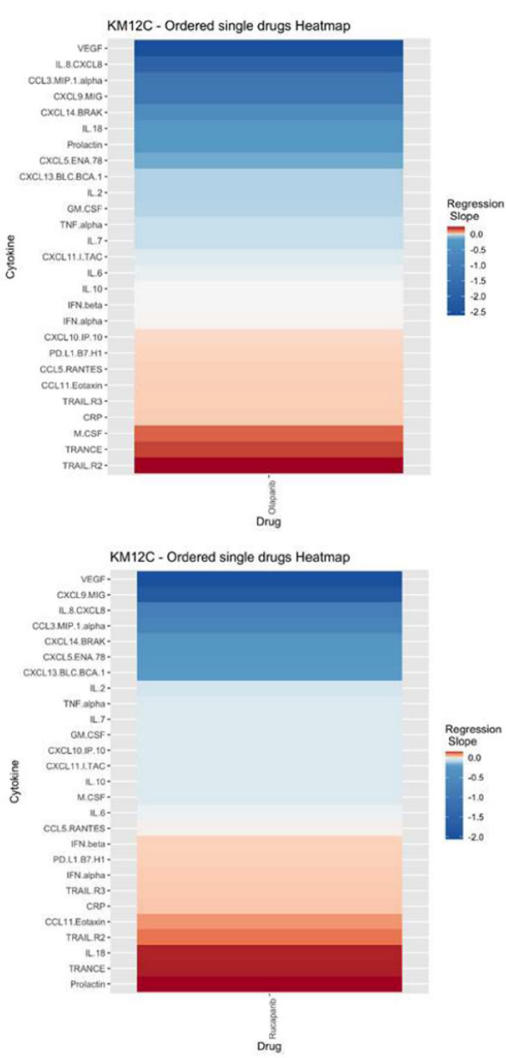

Figure 6: Heatmaps displaying regression slopes of cytokine profiles for PARP inhibitors. (A) Heat maps based on regression slopes for HCT-116, HT-29, and KM12C after 48-hour treatment of increasing doses of Olaparib or (B) Rucaparib. 
that can bind and prevent TRAIL-mediated apoptosis. To our knowledge, soluble TRAIL receptors have not yet been characterized as potential biomarkers of immune response to therapeutics in the context of cancer. These may be novel biomarkers for assessing the innate immune system as impacted by cancer therapeutics and would be especially relevant in the context of immunotherapies such as $\alpha$ PD- $1, \alpha$ PD-L1, and $\alpha$ CTLA4. Furthermore, these are relevant biomarkers in the context of TRAIL-receptor agonists such as ABBV-621, IGN-8444, INBRX-109, and AMG-655 which could be bound by sTRAIL-R2, potentially reducing therapeutic efficacy. However, the extent to which soluble TRAIL-R2/R3 can predict therapeutic efficacy in humans or in mice remains to be determined.

We also analyzed soluble receptor ligand programed-death ligand 1 (sPD-L1). PD-L1 is a transmembrane molecule that belongs to the B7 family and acts by binding to PD-1 on the surface of lymphocytes to inhibit the differentiation and proliferation of immune cells [45]. In CRC, elevated expression of PD-L1 is associated with poor prognosis, survival, and lymph node metastasis [45]. Furthermore, PD-L1 expression due to IFN- $\gamma$ signaling predicts poor survival in CRC [46]. PDL1 is thought to be most relevant as a biomarker in the context of immunotherapy, where many have described both predictive and prognostic roles of PD-L1 in colorectal cancer, and several other cancer types [47]. PD-L1 is currently being evaluated as a biomarker of poor prognosis in patients with CRC undergoing immunotherapy [47]. The soluble version of PD-L1, specifically, is an emerging biomarker of focus in $\mathrm{CRC}$ and increased sPD-L1 expression post-neoadjuvant chemoradiotherapy is correlated with worse disease-free survival [9].

We observed heterogeneity in cytokine, chemokine, and growth factor responses across cell lines and across drug treatments. When the results were grouped by either (1) analytes that are correlated with immunosuppression or
A

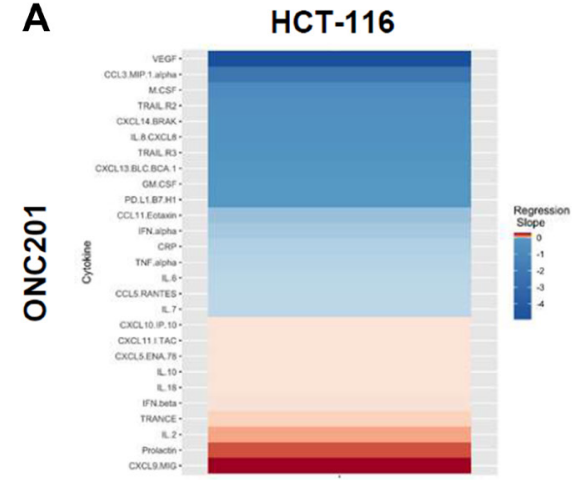

B

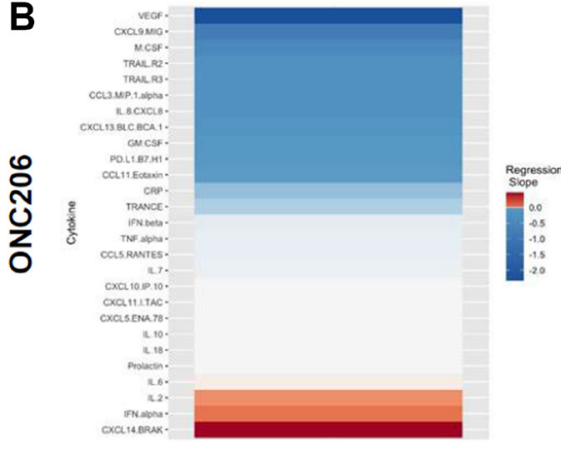

C

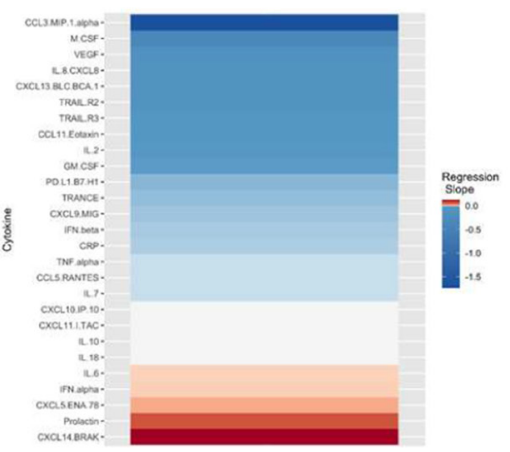

HT-29
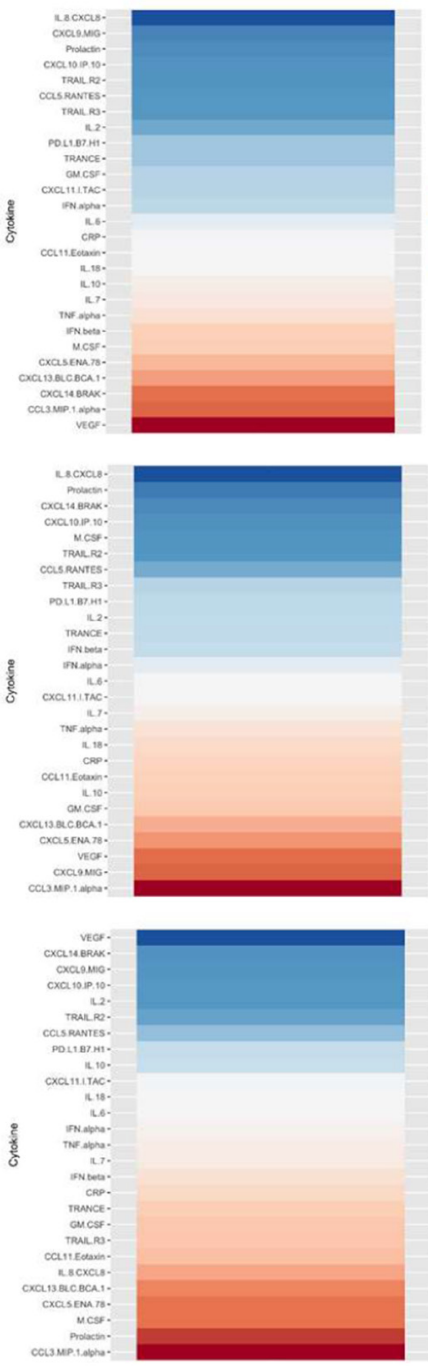

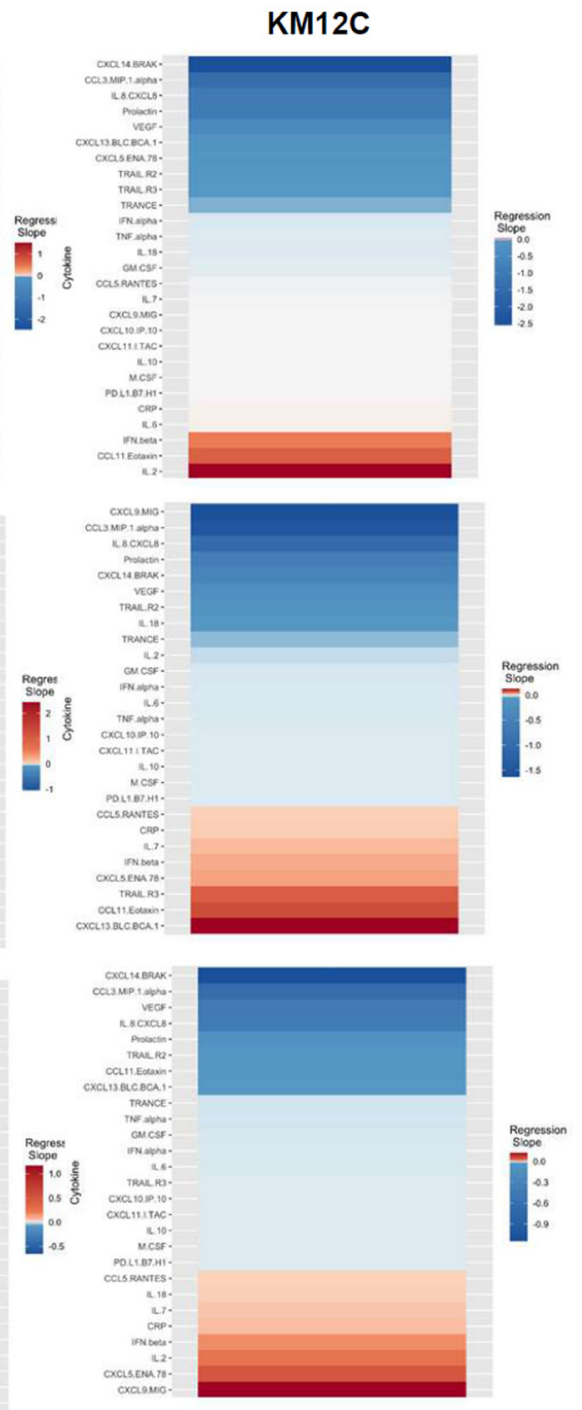

Figure 7: Heatmaps displaying regression slopes of cytokine profiles for Imipridones. (A) Heat maps based on regression slopes for HCT-116, HT-29, and KM12C after 48-hour treatment of increasing doses of ONC201, (B) ONC206, or (C) ONC212. 
unfavorable prognosis or by (2) analytes that are correlated with immunostimulation or favorable prognosis, we found that it was difficult to make clear predictions about which combinations of therapeutics would promote anti-tumor immunity in the context of CRC. This heterogeneous response to therapeutic treatment indicates that therapeutic immunomodulation is not necessarily predictable based on the tumor cytokinome alone. However, our dataset is limited and perhaps clearer trends would emerge with a greater sample size. Moreover, this dataset is amenable to building a larger database with other cytokinome data. We present our results as a novel platform with a large panel of relevant cytokines, chemokines, and growth factors that can impact therapeutic and immune response in the complex tumor microenvironment. Future studies can focus on specific cell lines, tumor types, classes of drugs, and subsets of cytokines. Additionally, we are currently pursuing the utilization of this platform for the investigation of drug combinations.

\section{MATERIALS AND METHODS}

\section{Cell culture}

Human colorectal cancer cells HCT-116, HT-29, and KM12C were used in this study. HCT-116 and HT-29 were cultured in McCoy's 5A (modified) Medium supplemented with $10 \%$ FBS and 1\% Penicillin-Streptomycin. KM12C cells were cultured in Eagle's Minimal Essential Medium Supplemented with 10\% FBS and 1\% PenicillinStreptomycin. All cell lines were incubated at $37^{\circ} \mathrm{C}$ in a humidified atmosphere containing $5 \% \mathrm{CO}_{2}$. Cell lines were authenticated and tested to ensure the cultures were free of mycoplasma infection.

\section{Measurement of cell viability}

Cells were plated at a density of $3 \times 10^{3}$ cells per well in a 96-well plate (Greiner Bio-One, Monroe, NC, USA). Cell viability was assessed using the CellTiter Glo assay (Promega, Madison, WI, USA). Cells were mixed with $25 \mu \mathrm{l}$ of CellTiter-Glo reagents in $100 \mu \mathrm{l}$ of culture volume, and bioluminescence imaging was measured using the Xenogen IVIS imager (Caliper Life Sciences, Waltham, MA, USA).

\section{Collection of culture supernatants used in cytokine measurements}

Cells were plated at $3.5 \times 10^{4}$ cells in a 48 well plate (Thermo Fisher Scientific, Waltham, MA, USA) in complete medium and incubated at $37^{\circ} \mathrm{C}$ with $5 \% \mathrm{CO}_{2}$. At 24 hours after plating, almost all the tumor cells were adherent to the bottom of the flask and the complete medium was replaced with drug-containing medium. Subsequently, the culture supernatants were collected after
48 hours of incubation and were frozen at $-80^{\circ} \mathrm{C}$ until the measurement of cytokines was performed. The day of analysis, samples were thawed and centrifuged to remove cellular debris.

\section{Cytokine, chemokine, and growth factor profiling}

An R\&D systems Human Premixed Multi-Analyte Kit (R\&D Systems, Inc., Minneapolis, MN) was run on a Luminex 200 Instrument (LX200-XPON-RUO, Luminex Corporation, Austin, TX) according to the manufacturer's instructions. Cell culture supernatant levels of TNF-alpha, IL-6, IL-8/CXCL8, Ferritin, IFN-beta, IL-10, CCL2/JE/ MCP-1, VEGF, CXCL13/BLC/BCA-1, IFN-gamma, CCL20/MIP-3 alpha, CCL3/MIP-1 alpha, CCL22/MDC, CCL4/MIP-1 beta, IL-4, IL-17/IL-17a, TRAIL R2/ TNFRSF10B, GM-CSF, CXCL5/ENA-78, CXCL9/MIG, G-CSF, CXCL11/I-TAC, Granzyme B, CCL5/RANTES, Prolactin, IFN-alpha, CXCL14/BRAK, IL-12/IL-23 p40, CXCL10/IP-10/CRG2, CCL7/MCP-3/MARC, IL7, CCL8/MCP-2, TRANCE/TNFSF11/RANK L, IL-15, TRAIL R3/TNFRSF10C, CCL11/Eotaxin, IL-18/IL-1F4, TRAIL/TNFSF10, IL-21, and C-Reactive Protein/CRP were measured.

\section{Bioinformatics analysis}

A quantitative analysis with 6 standards and a minimum of 50 counts per bead region was used with the Luminex to generate analyte values reported as picograms/ milliliter $(\mathrm{pg} / \mathrm{mL})$. Cytokine concentrations less than the lower limit of detection for each particular cytokine were recoded as zero. Cytokines without detectable expression levels were removed from further analysis of each cell line. Each drug was tested at different inhibitory concentrations (IC-10, IC-30, IC-50, IC-70, and IC-90), and these varying concentrations were used to measure a dose-response effect on cytokine expression. Cytokine dose-response effect was modeled by simple linear regression for each drug. The slopes of the linear regressions were compared. Data analysis and visualization were generated using R ( R Development Core Team, 2020).

\section{Author contributions}

K.H.- Conceptualization, Data curation, Formal analysis, Investigation, Methodology, Writing original draft, Writing - review and editing. A.L.Formal analysis, Investigation, Methodology, Writing - original draft, Writing - review and editing. L.Z.Conceptualization, Investigation, Methodology, Writing - original draft, Writing - review and editing. W.S.E-D.Conceptualization, Resources, Supervision, Funding acquisition, Investigation, Methodology, Writing - original draft, Project administration, Writing - review and editing. 


\section{ACKNOWLEDGMENTS}

W.S.E-D. is an American Cancer Society Research Professor and is supported by the Mencoff Family University Professorship at Brown University. This work was supported by an NIH grant (CA173453) to W.S.E-D. and by the Teymour Alireza P'98, P'00 Family Cancer Research Fund established by the Alireza Family. The contents of this manuscript are solely the responsibility of the authors and do not necessarily represent the official views of the National Cancer Institute, the National Institutes of Health, or the American Cancer Society.

\section{CONFLICTS OF INTEREST}

W.S.E-D. is a co-founder of Oncoceutics, Inc., a subsidiary of Chimerix. Dr. El-Deiry has disclosed his relationship with Oncoceutics and potential conflict of interest to his academic institution/employer and is fully compliant with NIH and institutional policy that is managing this potential conflict of interest.

\section{REFERENCES}

1. Turner MD, Nedjai B, Hurst $T$, Pennington DJ. Cytokines and chemokines: At the crossroads of cell signalling and inflammatory disease. Biochim Biophys Acta. 2014; 1843:2563-82. https://doi.org/10.1016/j. bbamcr.2014.05.014. [PubMed]

2. Berraondo P, Sanmamed MF, Ochoa MC, Etxeberria I, Aznar MA, Pérez-Gracia JL, Rodríguez-Ruiz ME, PonzSarvise M, Castañón E, Melero I. Cytokines in clinical cancer immunotherapy. Br J Cancer. 2019; 120:6-15. https://doi.org/10.1038/s41416-018-0328-y. [PubMed]

3. Jones VS, Huang RY, Chen LP, Chen ZS, Fu L, Huang RP. Cytokines in cancer drug resistance: Cues to new therapeutic strategies. Biochim Biophys Acta. 2016; 1865:255-65. https://doi.org/10.1016/j.bbcan.2016.03.005. [PubMed]

4. Yamaguchi $\mathrm{M}$, Okamura $\mathrm{S}$, Yamaji $\mathrm{T}$, Iwasaki $\mathrm{M}$, Tsugane S, Shetty V, Koizumi T. Plasma cytokine levels and the presence of colorectal cancer. PLoS One. 2019; 14:e0213602. https://doi.org/10.1371/journal. pone.0213602. [PubMed]

5. Gunawardene A, Dennett E, Larsen P. Prognostic value of multiple cytokine analysis in colorectal cancer: a systematic review. J Gastrointest Oncol. 2019; 10:134-43. https://doi. org/10.21037/jgo.2018.07.11. [PubMed]

6. Mager LF, Wasmer MH, Rau TT, Krebs P. CytokineInduced Modulation of Colorectal Cancer. Front Oncol. 2016; 6:96. https://doi.org/10.3389/fonc.2016.00096. [PubMed]

7. Ozaki K, Leonard WJ. Cytokine and cytokine receptor pleiotropy and redundancy. J Biol Chem. 2002; 277:2935558. https://doi.org/10.1074/jbc.R200003200. [PubMed]
8. Park JW, Chang HJ, Yeo HY, Han N, Kim BC, Kong SY, Kim J, Oh JH. The relationships between systemic cytokine profiles and inflammatory markers in colorectal cancer and the prognostic significance of these parameters. Br J Cancer. 2020; 123:610-18. https://doi.org/10.1038/s41416020-0924-5. [PubMed]

9. Tominaga T, Akiyoshi T, Yamamoto N, Taguchi S, Mori S, Nagasaki T, Fukunaga Y, Ueno M. Clinical significance of soluble programmed cell death-1 and soluble programmed cell death-ligand 1 in patients with locally advanced rectal cancer treated with neoadjuvant chemoradiotherapy. PLoS One. 2019; 14:e0212978. https://doi.org/10.1371/journal. pone.0212978. [PubMed]

10. Zhu Z, Zhang X, Guo H, Fu L, Pan G, Sun Y. CXCL13CXCR5 axis promotes the growth and invasion of colon cancer cells via PI3K/AKT pathway. Mol Cell Biochem. 2015; 400:287-95. https://doi.org/10.1007/s11010-0142285-y. [PubMed]

11. Cheadle EJ, Riyad K, Subar D, Rothwell DG, Ashton G, Batha H, Sherlock DJ, Hawkins RE, Gilham DE. Eotaxin-2 and colorectal cancer: a potential target for immune therapy. Clin Cancer Res. 2007; 13:5719-28. https://doi. org/10.1158/1078-0432.CCR-07-1145. [PubMed]

12. Fan F, Wey JS, McCarty MF, Belcheva A, Liu W, Bauer TW, Somcio RJ, Wu Y, Hooper A, Hicklin DJ, Ellis LM. Expression and function of vascular endothelial growth factor receptor-1 on human colorectal cancer cells. Oncogene. 2005; 24:2647-53. https://doi.org/10.1038/ sj.onc.1208246. [PubMed]

13. Chen G, Han G, Shen B, Li Y. GM-CSF facilitates the development of inflammation-associated colorectal carcinoma. Oncoimmunology. 2014; 3:e28186. https://doi. org/10.4161/onci.28186. [PubMed]

14. Al Obeed OA, Alkhayal KA, Al Sheikh A, Zubaidi AM, Vaali-Mohammed MA, Boushey R, Mckerrow JH, Abdulla $\mathrm{MH}$. Increased expression of tumor necrosis factor- $\alpha$ is associated with advanced colorectal cancer stages. World J Gastroenterol. 2014; 20:18390-96. https://doi.org/10.3748/ wig.v20.i48.18390. [PubMed]

15. Waldner MJ, Foersch S, Neurath MF. Interleukin-6--a key regulator of colorectal cancer development. Int J Biol Sci. 2012; 8:1248-53. https://doi.org/10.7150/ijbs.4614. [PubMed]

16. Neradugomma NK, Subramaniam D, Tawfik OW, Goffin V, Kumar TR, Jensen RA, Anant S. Prolactin signaling enhances colon cancer stemness by modulating Notch signaling in a Jak2-STAT3/ERK manner. Carcinogenesis. 2014; 35:795806. https://doi.org/10.1093/carcin/bgt379. [PubMed]

17. Mroczko B, Groblewska M, Wereszczyńska-Siemiatkowska U, Okulczyk B, Kedra B, Łaszewicz W, Dabrowski A, Szmitkowski M. Serum macrophage-colony stimulating factor levels in colorectal cancer patients correlate with lymph node metastasis and poor prognosis. Clin Chim Acta. 2007; 380:208-12. https://doi.org/10.1016/j. cca.2007.02.037. [PubMed] 
18. Zhao J, Ou B, Han D, Wang P, Zong Y, Zhu C, Liu D, Zheng M, Sun J, Feng H, Lu A. Tumor-derived CXCL5 promotes human colorectal cancer metastasis through activation of the ERK/Elk-1/Snail and AKT/GSK3 $\beta / \beta$-catenin pathways. Mol Cancer. 2017; 16:70. https://doi.org/10.1186/s12943017-0629-4. [PubMed]

19. Zumwalt TJ, Arnold M, Goel A, Boland CR. Active secretion of CXCL10 and CCL5 from colorectal cancer microenvironments associates with GranzymeB+ CD8+ T-cell infiltration. Oncotarget. 2015; 6:2981-91. https://doi. org/10.18632/oncotarget.3205. [PubMed]

20. Wang Y, Wang M, Li Y. Anti-colorectal cancer effect of interleukin-2 and interferon- $\beta$ fusion gene driven by carcinoembryonic antigen promoter. Onco Targets Ther. 2016; 9:3259-67. https://doi.org/10.2147/OTT.S97444. [PubMed]

21. Allen F, Bobanga ID, Rauhe P, Barkauskas D, Teich N, Tong C, Myers J, Huang AY. CCL3 augments tumor rejection and enhances $\mathrm{CD} 8+\mathrm{T}$ cell infiltration through NK and CD103+ dendritic cell recruitment via IFN $\gamma$. Oncoimmunology. 2017; 7:e1393598. https://doi.org/10.1 080/2162402X.2017.1393598. [PubMed]

22. Zhang R, Tian L, Chen LJ, Xiao F, Hou JM, Zhao X, Li G, Yao B, Wen YJ, Li J, Zhang L, Chen XC, Luo F, et al. Combination of MIG (CXCL9) chemokine gene therapy with low-dose cisplatin improves therapeutic efficacy against murine carcinoma. Gene Ther. 2006; 13:1263-71. https://doi.org/10.1038/sj.gt.3302756. [PubMed]

23. Cao Y, Jiao N, Sun T, Ma Y, Zhang X, Chen H, Hong J, Zhang Y. CXCL11 Correlates With Antitumor Immunity and an Improved Prognosis in Colon Cancer. Front Cell Dev Biol. 2021; 9:646252. https://doi.org/10.3389/ fcell.2021.646252. [PubMed]

24. Cao B, Yang Y, Pan Y, Jia Y, Brock MV, Herman JG, Guo M. Epigenetic silencing of CXCL14 induced colorectal cancer migration and invasion. Discov Med. 2013; 16:13747. [PubMed]

25. Maeurer MJ, Walter W, Martin D, Zitvogel L, Elder E, Storkus W, Lotze MT. Interleukin-7 (IL-7) in colorectal cancer: IL-7 is produced by tissues from colorectal cancer and promotes preferential expansion of tumour infiltrating lymphocytes. Scand J Immunol. 1997; 45:182-92. https:// doi.org/10.1046/j.1365-3083.1997.d01-384.x. [PubMed]

26. Lu C, Klement JD, Ibrahim ML, Xiao W, Redd PS, NayakKapoor A, Zhou G, Liu K. Type I interferon suppresses tumor growth through activating the STAT3-granzyme B pathway in tumor-infiltrating cytotoxic T lymphocytes. J Immunother Cancer. 2019; 7:157. https://doi.org/10.1186/ s40425-019-0635-8. [PubMed]

27. Cao D, Hou M, Guan YS, Jiang M, Yang Y, Gou HF. Expression of HIF-1alpha and VEGF in colorectal cancer: association with clinical outcomes and prognostic implications. BMC Cancer. 2009; 9:432. https://doi. org/10.1186/1471-2407-9-432. [PubMed]

28. Bendardaf R, Buhmeida A, Hilska M, Laato M, Syrjänen S, Syrjänen K, Collan Y, Pyrhönen S. VEGF-1 expression in colorectal cancer is associated with disease localization, stage, and long-term disease-specific survival. Anticancer Res. 2008; 28:3865-70. [PubMed]

29. Werther K, Christensen IJ, Nielsen HJ, and Danish RANX05 Colorectal Cancer Study Group. Prognostic impact of matched preoperative plasma and serum VEGF in patients with primary colorectal carcinoma. Br J Cancer. 2002; 86:417-23. https://doi.org/10.1038/sj.bjc.6600075. [PubMed]

30. Bestas R, Isikdogan A, Cil T, Batun S, Altintas A. The corelation between serum VEGF levels and known prognostic risk factors in colorectal carcinoma. J Clin Oncol. 2008; 26:S15070. https://doi.org/10.1200/ jco.2008.26.15_suppl.15070.

31. Kistner L, Doll D, Holtorf A, Nitsche U, Janssen KP. Interferon-inducible CXC-chemokines are crucial immune modulators and survival predictors in colorectal cancer. Oncotarget. 2017; 8:89998-90012. https://doi.org/10.18632/ oncotarget.21286. [PubMed]

32. Bie Y, Ge W, Yang Z, Cheng X, Zhao Z, Li S, Wang W, Wang Y, Zhao X, Yin Z, Li Y. The Crucial Role of CXCL8 and Its Receptors in Colorectal Liver Metastasis. Dis Markers. 2019; 2019:8023460. https://doi. org/10.1155/2019/8023460. [PubMed]

33. Shen T, Yang Z, Cheng X, Xiao Y, Yu K, Cai X, Xia C, Li Y. CXCL8 induces epithelial-mesenchymal transition in colon cancer cells via the PI3K/Akt/NF- $\mathrm{BB}$ signaling pathway. Oncol Rep. 2017; 37:2095-100. https://doi.org/10.3892/ or.2017.5453. [PubMed]

34. Allen F, Rauhe P, Askew D, Tong AA, Nthale J, Eid S, Myers JT, Tong C, Huang AY. CCL3 Enhances Antitumor Immune Priming in the Lymph Node via IFN $\gamma$ with Dependency on Natural Killer Cells. Front Immunol. 2017; 8:1390. https://doi.org/10.3389/fimmu.2017.01390. [PubMed]

35. Li X, Zhao L, Meng T. Upregulated CXCL14 is associated with poor survival outcomes and promotes ovarian cancer cells proliferation. Cell Biochem Funct. 2020; 38:613-20. https://doi.org/10.1002/cbf.3516. [PubMed]

36. Sjöberg E, Augsten M, Bergh J, Jirström K, Östman A. Expression of the chemokine CXCL14 in the tumour stroma is an independent marker of survival in breast cancer. $\mathrm{Br}$ J Cancer. 2016; 114:1117-24. https://doi.org/10.1038/ bjc.2016.104. [PubMed]

37. Westrich JA, Vermeer DW, Colbert PL, Spanos WC, Pyeon D. The multifarious roles of the chemokine CXCL14 in cancer progression and immune responses. Mol Carcinog. 2020; 59:794-806. https://doi.org/10.1002/mc.23188. [PubMed]

38. Zeng J, Yang X, Cheng L, Liu R, Lei Y, Dong D, Li F, Lau QC, Deng L, Nice EC, Xie K, Huang C. Chemokine CXCL14 is associated with prognosis in patients with colorectal carcinoma after curative resection. J Transl Med. 2013; 11:6. https://doi.org/10.1186/1479-5876-11-6. [PubMed] 
39. Lin K, Zou R, Lin F, Zheng S, Shen X, Xue X. Expression and effect of CXCL14 in colorectal carcinoma. Mol Med Rep. 2014; 10:1561-68. https://doi.org/10.3892/ mmr.2014.2343. [PubMed]

40. Soroush AR, Zadeh HM, Moemeni M, Shakiba B, Elmi S. Plasma prolactin in patients with colorectal cancer. BMC Cancer. 2004; 4:97. https://doi.org/10.1186/1471-2407-497. [PubMed]

41. Aldinucci D, Colombatti A. The inflammatory chemokine CCL5 and cancer progression. Mediators Inflamm. 2014; 2014:292376. https://doi.org/10.1155/2014/292376. [PubMed]

42. Chang LY, Lin YC, Mahalingam J, Huang CT, Chen TW, Kang CW, Peng HM, Chu YY, Chiang JM, Dutta A, Day YJ, Chen TC, Yeh CT, Lin CY. Tumor-derived chemokine CCL5 enhances TGF- $\beta$-mediated killing of CD8(+) T cells in colon cancer by T-regulatory cells. Cancer Res. 2012; 72:1092-102. https://doi.org/10.1158/0008-5472.CAN-112493. [PubMed]

43. Zhang S, Zhong M, Wang C, Xu Y, Gao WQ, Zhang Y. CCL5-deficiency enhances intratumoral infiltration of CD8+ T cells in colorectal cancer. Cell Death Dis. 2018; 9:766. https://doi.org/10.1038/s41419-018-0796-2. [PubMed]
44. Chen C, Xu ZQ, Zong YP, Ou BC, Shen XH, Feng H, Zheng $\mathrm{MH}$, Zhao JK, Lu AG. CXCL5 induces tumor angiogenesis via enhancing the expression of FOXD1 mediated by the AKT/NF- $\kappa$ B pathway in colorectal cancer. Cell Death Dis. 2019; 10:178. https://doi.org/10.1038/s41419-019-1431-6. [PubMed]

45. Shan $\mathrm{T}$, Chen $\mathrm{S}, \mathrm{Wu} \mathrm{T}$, Yang Y, Li S, Chen X. PD-L1 expression in colon cancer and its relationship with clinical prognosis. Int J Clin Exp Pathol. 2019; 12:1764-69. [PubMed]

46. Zhao T, Li Y, Zhang J, Zhang B. PD-L1 expression increased by IFN- $\gamma$ via JAK2-STAT1 signaling and predicts a poor survival in colorectal cancer. Oncol Lett. 2020; 20:1127-34. https://doi.org/10.3892/o1.2020.11647. [PubMed]

47. Li Y, He M, Zhou Y, Yang C, Wei S, Bian X, Christopher O, Xie L. The Prognostic and Clinicopathological Roles of PDL1 Expression in Colorectal Cancer: A Systematic Review and Meta-Analysis. Front Pharmacol. 2019; 10:139. https:// doi.org/10.3389/fphar.2019.00139. [PubMed] 